\title{
Mobile Intelligence Architecture
}

\author{
P.Manikantan \\ Ph.D - Research Scholar \\ Vinayaka Mission University, \\ Salem, Tamilnadu, India
}

\author{
Dr.M.V.Srinath, Ph.D \\ Head- ID, \\ Hi-Command Tech India Ltd, \\ Tamilnadu, India
}

\author{
Dr.G.M.Kadhar Nawaz \\ Ph.D \\ Director, Department of \\ Computer Science \\ Sona College of Technology, \\ Salem, Tamilnadu,India
}

\begin{abstract}
Artificial intelligence with Human-computer interaction is a discipline concerned with the design, evaluation and implementation of interactive computing systems for human use and with the study of major phenomena surrounding them. Current mobile computing devices such as palmtop computers, personal digital assistants (PDAs) and mobile phones, and future devices such as Blue tooth and GSM enabled cameras, and music players enabled have many implications for the design of the user interface. No longer can designers base computing designs around the traditional model of a single user working with a personal computer at his/her workplace. In addition to mobility and size requirements, mobile devices will also typically be used by a larger population spread than traditional PCs and without any training or support networks, whether formal or informal. Furthermore, unlike early computers which had many users per computer, and PCs with usually one computer per user, a single user is likely to own many mobiles devices which they interact with indifferent ways and for different tasks. Mobile devices present human-computer interaction (HCI).Using Artificial Intelligence Multitasking and support for task interruption is one of the keys to successful Synthesis Based Mobile Architecture.
\end{abstract}

\section{General Terms}

Artificial Intelligence, Machine Learning, Mobile Intelligence.

\section{Keywords}

Artificial Intelligence, Mobile Architecture, Excogitator,

Text-to-Speech, Auto Learner

\section{INTRODUCTION}

Human-computer interaction (HCI) or, alternatively, man machine interaction (MMI) or computer -human interaction is the study of interaction between people normally users and computers. It is an interdisciplinary subject, relating computer science with many other fields of study and research. Interaction between users and computers occurs at the user interface, which includes both software and hardware. The mobile phone is always carried with the user and is always active: it is a very personal device. It fosters and satisfies a need to be constantly connected to one's significant other, friends or business partners. The Synthesis Based Mobile Architecture framework assumes that mobility is inherently multitasking with fragmentary theory of intelligent reasoning, expressed and easily leads to competition for cognitive with mobile based human computer interaction. Excogitator is artificial intelligence software that learns automatically from the outside environment and performs all the activity requested by the user, automatically. The framework works by taking the meaning of words into account. The fact files contain information about anything. The excogitator will make construct the fact (information) by user input or automatically from external resources with human computer interaction. Excogitator is artificial intelligence software which also interacts with human that learns automatically from outside environment and performs all the activity requested by the user, automatically. Synthesis based mobile architecture works by breaking a user's message down into individual words and then searching through a list of facts for a fact that contains similar words, the facts with the most matching words is used as a response to the user's message and learning automatically from television, radio and internet and acknowledge to perform the task. Excogitator works by asking the meaning of words into account, it uses a large table of 'match words' to help it decide which words match or have similar meaning.

\subsection{Natural Language Processing}

Natural language [15] is only one medium for human machine interaction, but has several obvious and desirable properties which provides an immediate vocabulary for talking about the contents of the computer, It provides a means of accessing information in the computer (Fig 1) independently of its structure and encoding and It shields the user from the formal access language of the underlying system. In Human-Computer Interaction which via Mobile device towards interaction for device has virtual intelligence y commanding the mobile device to perform the task by the intelligence. The excogitator which normally device inclination software towards perform the task with intelligence.

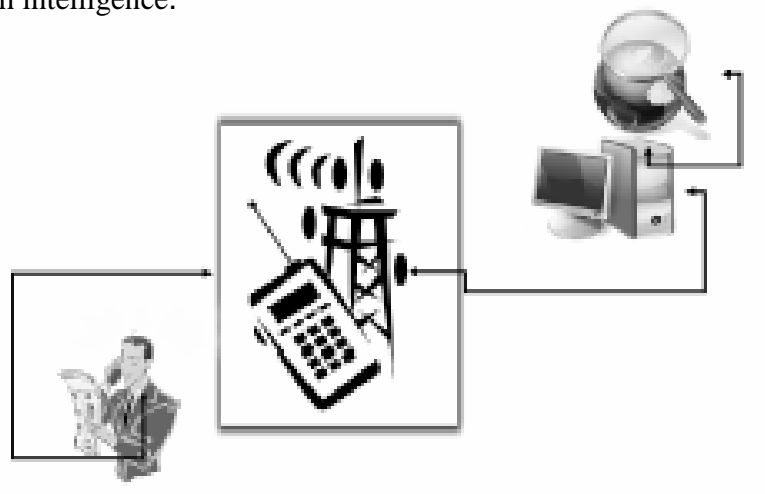

Fig1: Natural Language processing over mobile technology

\subsection{Grammar}

A grammar of a language [11] is a scheme for specifying the sentences in that language. It indicates the syntactic rules for combining words into well -formed phrases and clauses. The 
theory of generative grammar had a profound effect on linguistic research, including AI work in computational linguistics. In the excogitator architecture which include the English grammar to interact with end -user.

\subsection{Parsing}

Parsing is the "de-linearization" of linguistic input; that is, the use of grammatical rules [12] and other knowledge sources to determine the functions of words in the input sentence. Usually a parser produces a data structure like a derivation tree to represent the structural meaning of a sentence.

\subsection{Artificial Intelligence}

Artificial intelligence (AI) [2] is a broad field it has one of the concerned with getting computers to do tasks that require human intelligence. However, having said that, there are many tasks, which we might reasonably think require intelligence - such as complex arithmetic - which computers can do very easily with research was placed upon creating systems that could efficiently solve problems, by limiting the search, such as the logic theorist and second, making systems that could learn by themselves. Conversely, there are many asks that people do without even thinking. Up -to-the-minute technology mobile device which utilized and interaction by human for vast application. People might want to automate human intelligence for a number of different reasons. One reason is simply to understand human intelligence better. Synthesis based mobile architecture framework and application are representing knowledge. Architecture will be use the technique of representation of the mind-data will be processed and it learn from the outside environment and internal. Artificial intelligence research makes the assumption that human intelligence can be reduced to the (complex) manipulation of symbols, and that it does not matter what medium is used to manipulate these symbols - it does not have to be a biological brain. This assumption does not go unchallenged among philosophers etc. Some argue that true intelligence can never be achieved by a computer, but requires some human property which cannot be simulated.

\section{CONCEPTUAL DEPENDENCY}

Conceptual dependency as a representation for the meanings of phrases and sentences. For any two sentences that are identical in meaning, regardless of language, there should be only one representation of the meaning in $\mathrm{CD}$. Here interaction via mobile device and operate the computer to perform the task with intelligence. The excogitator which perform agent of the computer and device to promote the ask. In conceptual dependency, syntactic and semantic knowledge are combined into a single interpretation system that is driven by semantic knowledge. Syntactic parsing is subordinated to semantic interpretation usually provides a greater degree of predictive power.

\subsection{Semantic Grammar}

Semantic grammars combine syntactic, semantic, and pragmatic knowledge [15] into a single set of rules in the form of a grammar. This is usually just a context -free grammar in which the choice of non -terminal and production rules is governed by semantic as well as syntactic function. This is usually good for restricted natural -language interfaces

\subsection{Systemic Grammar}

Systemic grammars distinguish three general functions of language, all of which are ordinarily served by every act of speech.

\subsection{Machine Translation}

A proposed approach for translating between arbitrary languages was to go through an intermediate "universal language" or Interlingua. However, without commonsense world knowledge, certain kinds of translations are impossible. Simply using dictionaries doesn't solve the problem. The other troubles are Many words have several translations depending on context, and some don't have any (e.g., Japanese has two different words, "hot water" and "cold water"). The constructive [8] will understand the word by word. Word orders vary from language to language.

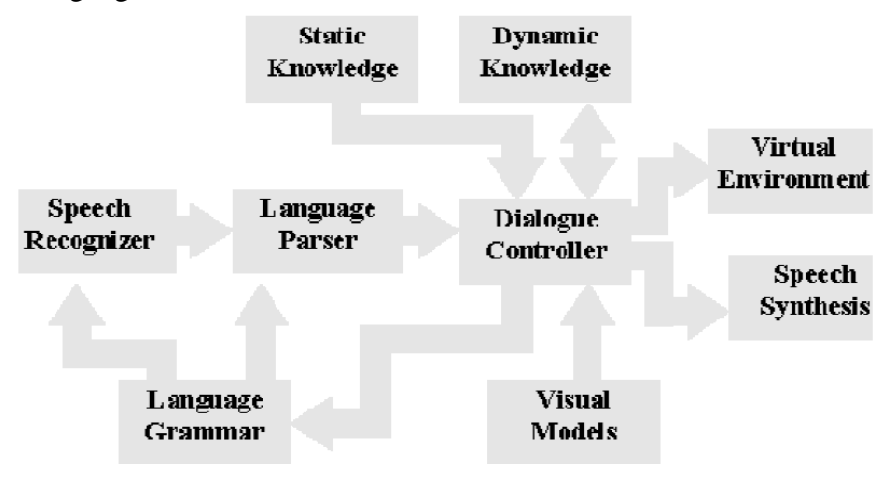

Fig 2: Natural Language Processing Transaction

Idiomatic expressions cannot be translated word -for-word must be translated in to real meaning to proceed by acknowledgement. $\mathrm{n}$ excogitator we used the technology of second type of speech service provides the ability to convert written text into spoken words. This is called text -to-speech (Fig 2) (TTS) technology [15]. Just as there are a number of factors to consider when developing speech recognition engines (SR), there are a few issues that must be addressed when creating and implementing rules for TTS engines. This speech engine gives more effective for speech reorganization.

\section{SEARCH TECHNIQUE}

Let's say that the "nodes" (e.g., a1, a2) represent towns and the links represent roads between the towns. Or simplicity we'll assume that these are all one -way streets and we've deliberately avoided loops, where you can back to where you were before by going round in a circle. So, the map is in fact a tree. Suppose we want to find a possible route from a star to state in town a1 to a goal state in town a6. we can quickly see that the only route involves going via a4 and a5 - but how do we systematically search for such a route. Here the mobile device interaction which need to the interaction which convert as command and need search the successful command to perform. The excogitator which perform the search of data for perform and completion of the task and acknowledge to the user depend success or failure. 


\subsection{Best First Search}

Best first search is a little like hill climbing, in that it uses an evaluation function and always chooses the next node to be that with the best score. However, it is exhaustive, in that it should eventually try all possible paths. It uses an agenda as in breadth/depth first search (Fig 3), but instead of taking the first node off the agenda and generating its successors. it will take the best node off, i.e. the node with the best score. The successors of the best node will be evaluated and added to the list. Here the best first search which perform the operation human-computer interaction[5] search of command and data or information toward perform users task with intelligence and short timing and powerful search technique. Best -First search become an efficient technique for the knowledge based constructive and it containing almost effective search data.

\subsection{Excogitator Architecture}

The Excogitator architecture based on an artificial intelligence for the purpose it to understand and respond to natural language programmed to take advantage of text-to-speech, speech recognition on human computer interaction (HCI) with Mobile architecture (Fig2) and can be used for many things; I can be used as an intelligent data bank or as a technical support tool. It would also make a good companion to an encyclopedia. Excogitator which designed with mobile technology and can memorize [5] Information that you interact and then when you ask any question and will try to answer using the information within my memory and information from outside the databank [6]. Excogitator architecture not only does what you are tried to find answers to your questions; It will also try to find and respond with information that is relevant to your statements and perform your manageable tasks. Excogitator is artificial intelligence software that learns automatically from the outside environment and performs all the activity requested by the user, automatically. The framework works by taking the meaning of words into account. The fact files contain information about anything. The excogitator will make construct the fact (information) by user input or automatically from external resources with human computer interaction. Excogitator is artificial intelligence software which also interacts with human that learns automatically from outside environment and performs all the activity requested by the user, automatically.

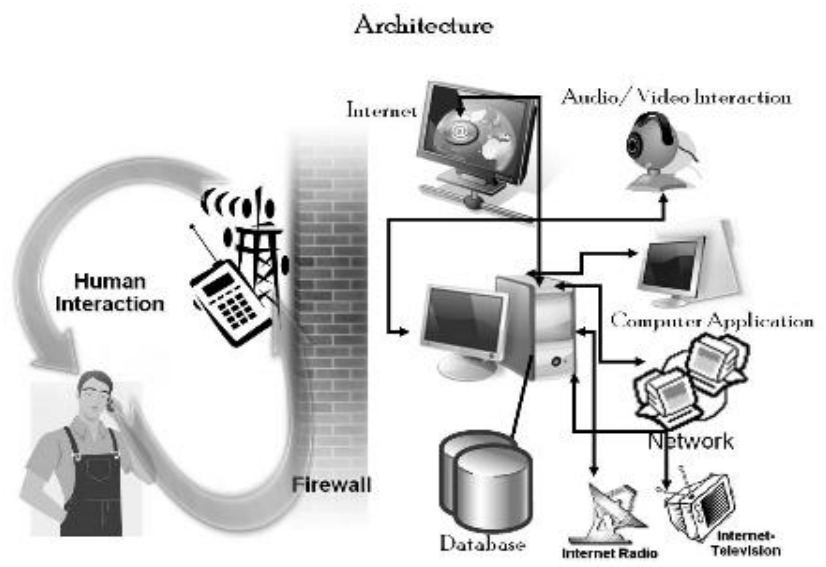

Fig3: Excogitator Interaction

\section{KNOWLEDGE REPRESENTATION}

Excogitator Knowledge representations which represent any intelligent [13] entity that wishes to reason about its world encounters an important, inescapable [11] fact: reasoning is a process that goes on internally, while most things it wishes to reason about exist only externally and representations function as surrogates for abstract notions like actions, processes, beliefs, causality, categories, etc., allowing them to be described inside an entity so it can reas on about them. Second, formal objects can of course exist inside the machine with perfect fidelity: Mathematical entities [3], for example, can be captured exactly, precisely because they are formal objects. Since almost any reasoning task will encounter $\mathrm{t}$ he need to deal with natural objects.

\subsection{Data Representation}

The excogitator architecture which represent the data with very large database collection of random work activation of 13 million random work can represent depend up on the capability of the user device and provide source to learn and merge the data constructively and productively increate the optimization for the effective search and utilize the data for extraction

\section{CONCLUSION AND FUTURE WORK}

This paper firstly introduced the definition of context, which has been considered as excogitator and knowledge representation by information in design, and positioned context as core information for a foundation for human computer interaction centered design methodology. Secondly, a general framework for representing and incorporating it in design information as forms of models was constructed using MMI as an underlying mechanism. Thirdly, auto -lean process mode methods were demonstrated to identify various contexts and their relations to other information elements and to perform the task. Although a general framework approach to the development of the representational mechanisms for contextual information was explained, further formal and empirical research is needed. Use of this architecture will provides facility in many different application domains opens a huge new space for research and system development including changes in mobile architecture with re-configurable interfaces. Further research progress to make the artificial brain chip for make faster computation with intelligence.

\section{ACKNOWLEDGMENTS}

An endeavor over a long period will be successful only with the advice and support of many well -wishers. It is the blissful chance bestowed upon me by the heaven. First, I would like to thank my parents and almighty for giving me courage and ability to take this venture. I would like to express my profound gratitude to my research advisor and appreciation is extended to ${ }^{22]}$ Dr.M.V.Srinath, Ph.D., - Head-ID, Hi-Commands Tech India Ltd, and Dr.G.M.Kadhar Nawaz Ph.D , Director, Department of Computer Science, Sona College of Technology. 


\section{REFERENCES}

[1] Artificial intelligence, by the editors of time -life books, Alexandra Virginia,ISBN-0-8094-5675-3

[2] Allen J., Hunnicutt S., and Klatt D. From text to speech: the MITalk system. MIT Press, Cambridge, MA,1987.

[3] Bailly G. and Benoit C., editors. Talking Machines: Theories,Models, and Designs. Elsevier Science, 1992.

[4] Donovan R.E. and Woodland P.C. "Improvements in an HMMBased Speech Synthesizer". Proceedings of Eurospeech Conference,Madrid, Spain, 1995, pages 573.

[5] Fernando Pereira, Michael Riley, and Richard Sproat.Weighted rational transductions and their application to hu-man language processing. In ARPA Workshop on Human Language Technology, pages 249254. Advanced Research Projects Agency, March 8 -11 1994.

[6] Hauptmann, A.G. and Smith, M.A. Te xt, Speech and Vision for Video Segmentation: the Informedia Project.AAAI Fall Symposium on Computational Models for Integrating Language and Vision, Boston MA Nov 10-12 1995.

[7] Hauptmann, A.G. and Witbrock, M.J., Informedia News on Demand:Multimedia Information Acquisition and Retrieval, in Maybury M.T., Ed, Intelligent Multimedia
Information Retrieval, AAAI Press/MIT Press, Menlo Park, 1996 (In Press).

[8] Julia Hirschberg. Pitch accent in context: Predicting international prominence from text. Artificial Intelligence, 63:305-340, 1993.

[9] Lauri Karttunen. Finite-state lexicon compiler. Technical Report P93-00077, Xerox Palo Alto Research Center, 1993.

[10] Lauri Karttunen and Kenneth Beesley. Two -level rule compiler.Technical Report P92-00149, Xerox Palo Alto Research Center,1992. [11] Microsoft Research's Speech Technology Group.

[12] Ronald Kaplan and Martin Kay. Regular models of phonological rule systems. Computational Linguistics,20:331 378,1994 .

[13] Schank, Roger, and Larry Hunter, " The Quest of Understand thinking” BYTE, April 1985

[14] Sproat R., Hirschberg J., and Yarowsky D. "A corpus-based synthesizer". International Conference on Spoken Language Systems, Banff, Canada, 1992, pages 563-566.

[15] Van Coile B. "On the Development of Pronunciation Rules for Textto-Speech Synthesis". Proceedings of Eurospeech Conference. 